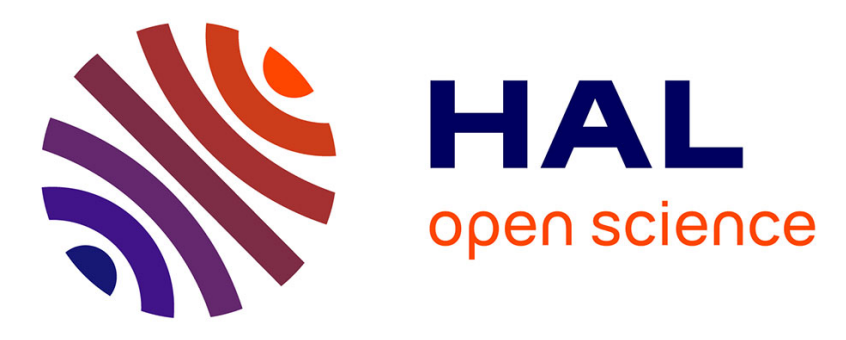

\title{
Topological Characteristics of Digital Models of Geological Core
}

\author{
Rustem R. Gilmanov, Alexander V. Kalyuzhnyuk, Iskander A. Taimanov, \\ Andrey A. Yakovlev
}

\section{- To cite this version:}

Rustem R. Gilmanov, Alexander V. Kalyuzhnyuk, Iskander A. Taimanov, Andrey A. Yakovlev. Topological Characteristics of Digital Models of Geological Core. 2nd International Cross-Domain Conference for Machine Learning and Knowledge Extraction (CD-MAKE), Aug 2018, Hamburg, Germany. pp.273-281, 10.1007/978-3-319-99740-7_19 . hal-02060056

\section{HAL Id: hal-02060056 https://hal.inria.fr/hal-02060056}

Submitted on 7 Mar 2019

HAL is a multi-disciplinary open access archive for the deposit and dissemination of scientific research documents, whether they are published or not. The documents may come from teaching and research institutions in France or abroad, or from public or private research centers.
L'archive ouverte pluridisciplinaire HAL, est destinée au dépôt et à la diffusion de documents scientifiques de niveau recherche, publiés ou non, émanant des établissements d'enseignement et de recherche français ou étrangers, des laboratoires publics ou privés.

\section{(c)(1)}

Distributed under a Creative Commons Attribution| 4.0 International License 


\title{
Topological Characteristics of digital models of geological core
}

\author{
R.R. Gilmanov ${ }^{1}$, A.V. Kalyuzhnyuk ${ }^{2}$, I.A. Taimanov ${ }^{3,4}$, and A.A. Yakovlev ${ }^{1}$ \\ ${ }^{1}$ OOO “Gazpromneft NTC”, 190000 St. Petersburg, Russia \\ \{Gilmanov.RR, Yakovlev.AAle\} @gazpromneft-ntc.ru \\ ${ }^{2}$ Peter the Great St.Petersburg Polytechnic University, 195251 St. Petersburg, Russia \\ Kalyuzhnyuk. AVegazprom-neft.ru \\ ${ }^{3}$ Chebyshev Laboratory at St. Petersburg State University, 199178 St. Petersburg, Russia \\ ${ }^{4}$ Sobolev Institute of Mathematics, 630090 Novosibirsk, Russia \\ taimanovemath.nsc.ru
}

\begin{abstract}
We discuss the possibility of applying stochastic approaches to core modeling by using tools of topology. The study demonstrates the prospects of applying topological characteristics for the description of the core and the search for its analogs. Moreover application of topological characteristics (for example, in conjunction with machine learning methods) in the long term will make it possible to obtain petrophysical properties of the core samples without carrying out expensive and long-term filtration experiments.
\end{abstract}

Keywords: Topological characteristics, Betti numbers, digital core, geological modeling.

Nowadays the process of oil fields exploitation needs a continuous information support. This is especially necessary in the context of the emphasis shift in the development, planning and monitoring of oil and gas fields on very highly dissected and low-permeability reservoirs.

More accurate estimation of economic efficiency and optimal placement of production wells are possible if we have geological picture of the oil field. This means that mathematical measure of the geological modeling of such objects is needed.

These estimation processes include a geological and hydrodynamic modeling. Adjustment of models includes:

- static well data - logging curves, core analysis, drilling data etc.;

- dynamic data - well flow, bottomhole pressure etc.

In practice, the adjustment of models occurs iteratively - by the numerical simulation of series of direct resource-intensive tasks. The speed of such adjustment depends on geological model quality. To measure quality of such model, a mathematical method is needed to describe its "heterogeneity" and "internal complexity". It is necessary to find a proper cell size, variogram radius, experimental data correlation etc. 
Similar problems also exist in the digital modeling of core samples - rock samples extracted from the well. In modern practice, there are some tools for measuring of the heterogeneity of such models [1]:

- construction of dissect's maps;

- spectral modeling of logging curves.

However, these methods do not provide numerical characteristics (metric, measure) of a constructed 3-D model. In [2,3] it was proposed to consider topological characteristics of these models. Here we use this approach to study digital models of geological core sample. The topological characteristics of core samples are compared with the topological characteristics of geological models.

\section{Topological characteristics of three-dimensional digital solid body models}

Topological characteristics of three-dimensional digital solid body models in the study are defined with the same mathematical apparatus as in [3]. These models represent ordered sets of elementary cubes - cubic complexes. The solid bodies are nottopologically equivalent if their Betti numbers $\boldsymbol{b}_{\boldsymbol{0}}, \boldsymbol{b}_{\boldsymbol{1}}$ and $\boldsymbol{b}_{2}$ are different. The meanings of these topological characteristics are follows:

- $\boldsymbol{b}_{\boldsymbol{0}}$ is the number of connected components;

- $\boldsymbol{b}_{\boldsymbol{1}}$ is the number of handles;

- $\boldsymbol{b}_{2}$ is the number of holes (cavities).

In this paper, two elementary cubes are considered to belong to the same linear component only in case of intersecting each other by a joint face. Intersecting by a joint vertex or edge does not make them belong to the same linear component.

If we start from a solid cube, remove $\boldsymbol{k}$ holes from its interior and attach $\boldsymbol{l}$ handles to the cube we obtain the body X for which $\boldsymbol{b}_{0}=1, \boldsymbol{b}_{1}=\boldsymbol{l}, \boldsymbol{b}_{2}=\boldsymbol{k}$.

Calculation of the Betti numbers for these cubic complexes was implemented via the numerical algorithm from [3].

\section{Digital core model analysis}

\subsection{Digital core model description}

The digital core model is a model obtained as a result of computer tomography. Method is based on computer-processed combinations of many X-ray measurements taken from various angles to produce cross-sectional images of a scanned object, allowing to see inside the object without destruction [4]. Today, computed tomography is an evolving method for studying the petrographic properties of rocks. The X-ray tomography method allows to solve a huge number of geological problems, such as 
modeling cavities (fracture, caverns, pores), calculating the porosity, studying rock heterogeneity, analysis of reservoir properties, measurement as core`s volumes as all its voids and solids.

X-ray tomography has been used in oil industry since 80-s years [5]. The first studies has been conducted in Australia, USA, and Great Britain [6].

The result of the X-ray tomography of a core sample is a set of grayscale snapshots representing corresponding virtual sections [7]. Each snapshot point shows its radiodensity. A combination of these grayscale snapshots is used then to generate 3dimensional radiodensity distribution of the sample in the volume [8].

\subsection{Digital core model creation process}

A core sample with a diameter of $46 \mathrm{~mm}$ and $7.8 \mathrm{~mm}$ high was used in the study.

Tomography snapshots are taken with $0.025 \mathrm{~mm}$ interval for inline and crossline sections and with $0.004 \mathrm{~mm}$ interval for vertical sections.

After the tomography scan, the result was stored in the SEG-Y format, commonly used to encode the results of seismic studies. This format is convenient for further analysis, because it permits to import the results in the form of voxels: a threedimensional array, where each element corresponds to the value of the radiodensity. (see. Hounsfield scale [9])

The value of radiodensity was taken in conventional units of radiodensity - certain number given by the device for computed tomography.

The core sample was imported into the Petrel (Schlumberger's oil engineering software package) (see Fig. 1). SEG-Y format is also useful to show virtual crosssections of a digital via Petrel (see Fig. 2). 


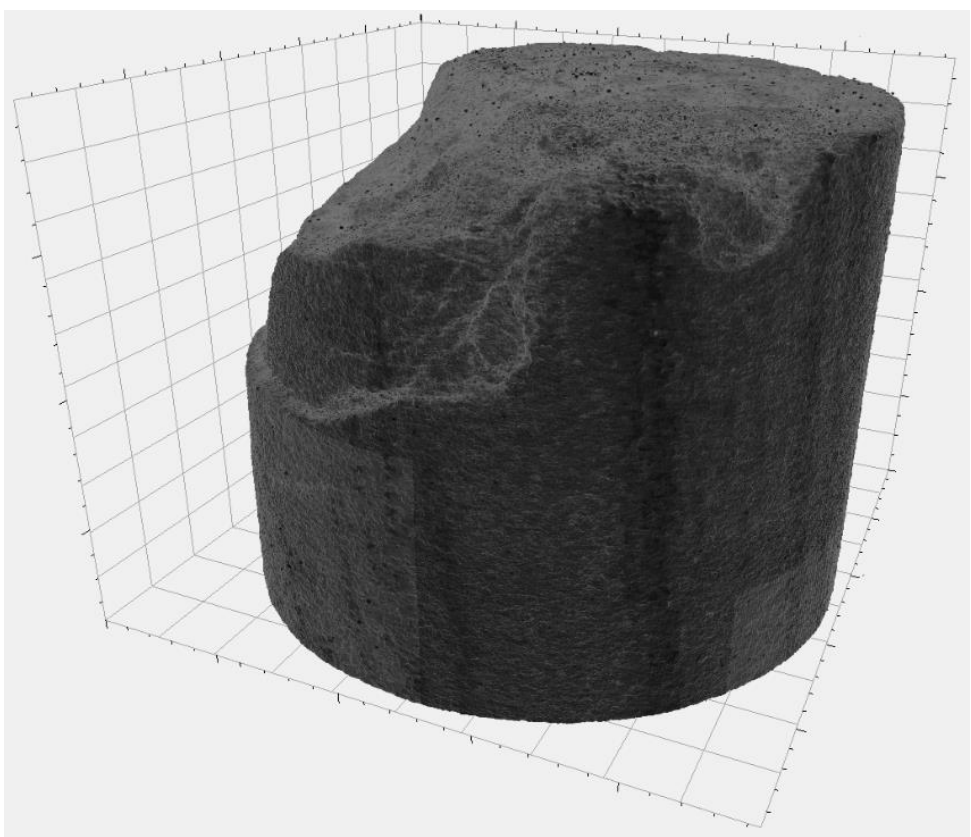

Fig. 1. Terrigenous core sample - 3D view of the sample (vertical axe is scaled).

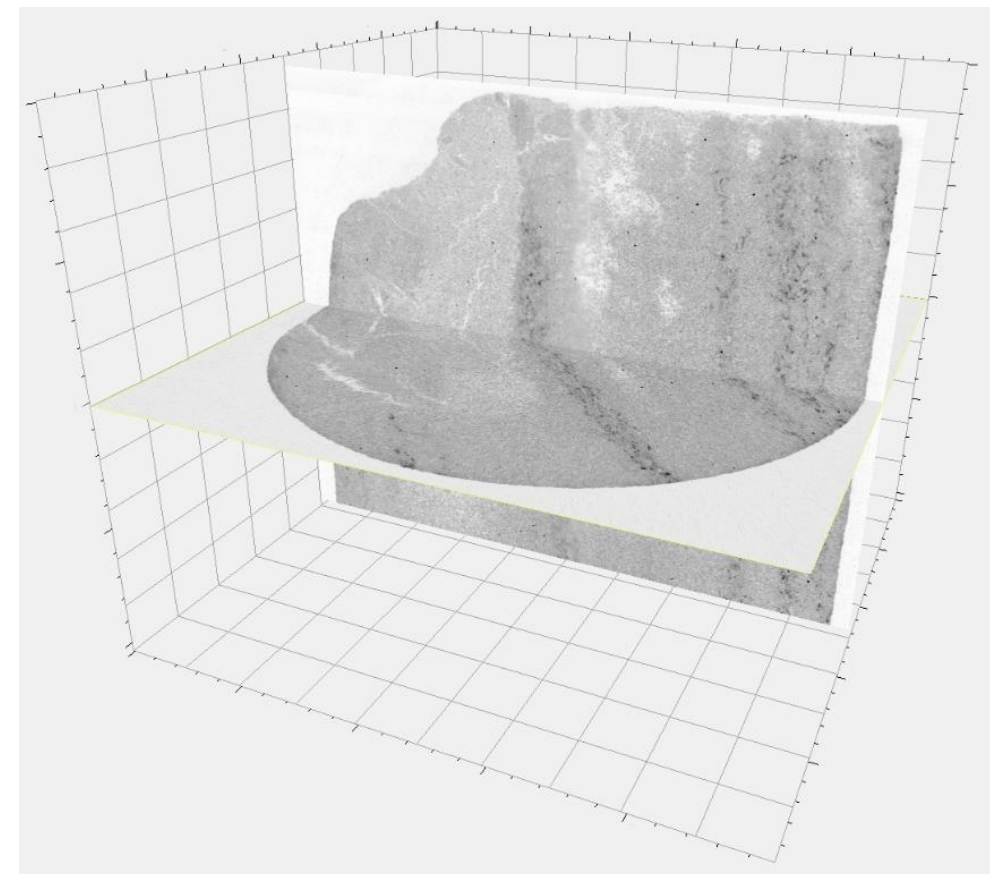

Fig. 2. . Terrigenous core sample inline cross-section (vertical axe is scaled). 


\subsection{Digital core model processing}

Three 3D cube-samples with the size of $100 \times 100 \times 100$ voxels are cut from the core model (Fig.2). The principle of "indicator formalism" is applied to the radiodensity values contained in voxels of cut cubes [10]. The range of voxel values is linearly mapped to the segment $[0,1]$, so that the smallest of the radiodensity values is mapped to 0 , the largest value is set to 1 . These values are hereinafter referred to as "normalized radiodensity".

These cube-samples are transformed to a .grdecl format to evaluate their topological characteristics and to a .vtk format to visualize them using the Paraview software.

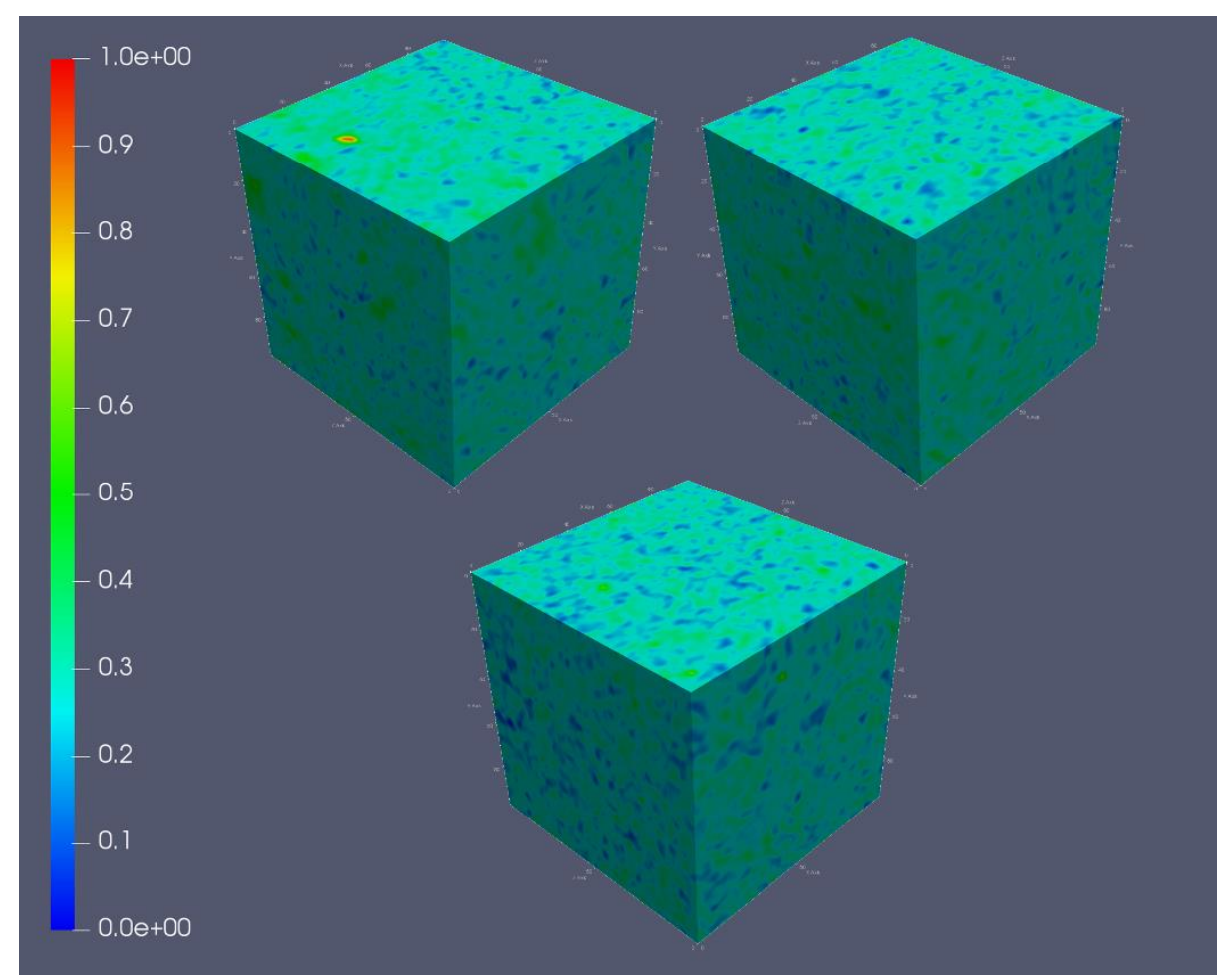

Fig. 3. Voxel cubes cut from the digital core model to calculating topological invariants (clockwise - 1st, 2nd and 3rd cube).

To calculate the model's topological characteristics, it is necessary to classify voxels to divide them according to the "skeleton of the rock" - "void". Voxels, marked as "skeleton of the rock", are then considered equivalent to elementary cubes from item 1.

The built-in material classifier of the tomography apparatus is not used in the study. For our purpose, we used an excursion parameter $\boldsymbol{\alpha}$, the value of which varies from 0 to 1 [3] (Fig.3). 
The application of the excursion parameter $\boldsymbol{\alpha}$ to the voxel model generates a cubic complex, where an elementary cube is a voxel whose normalized radiodensity value is greater than $\boldsymbol{\alpha}$.

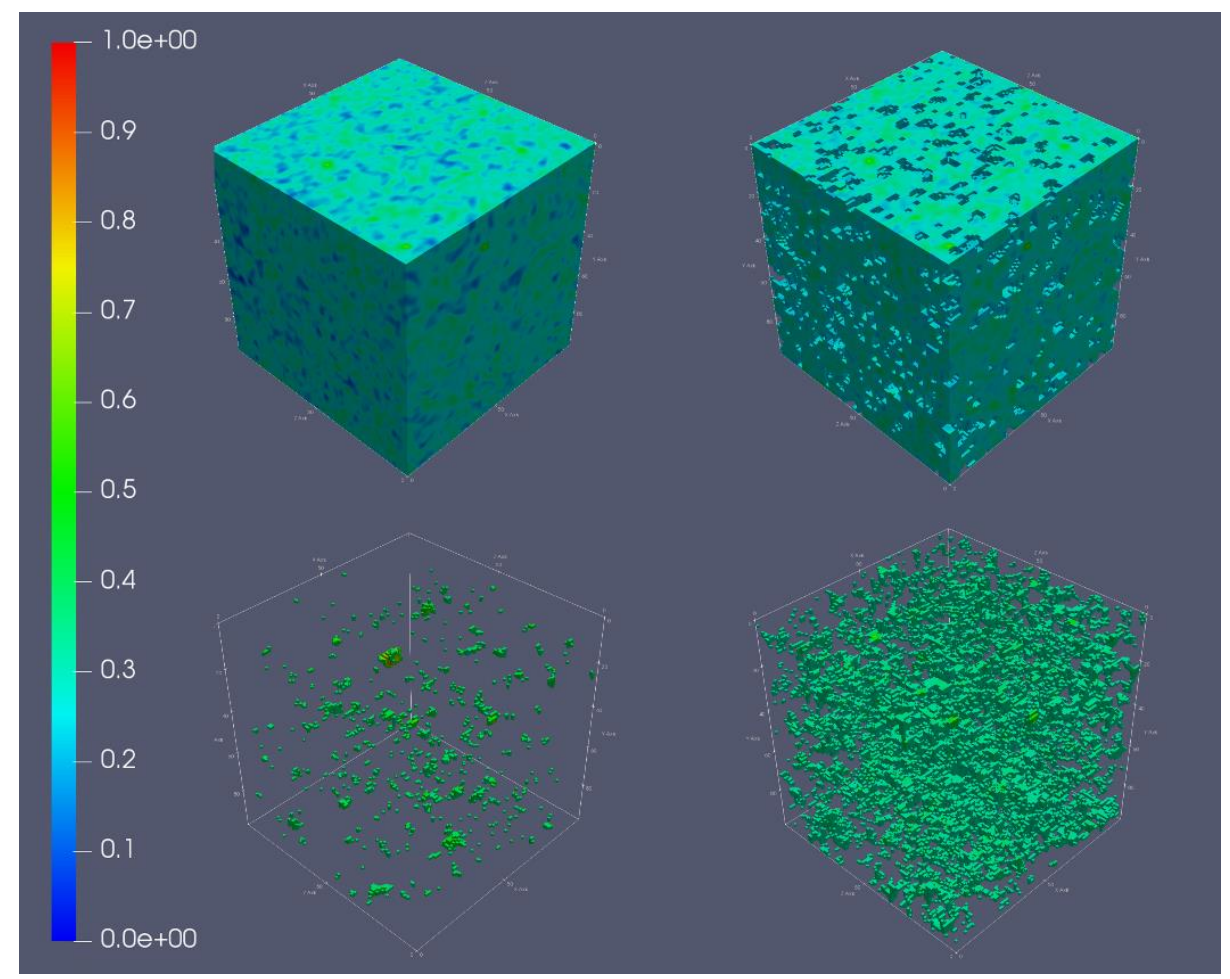

Fig. 4. An example of a core model separation of the "rock skeleton" - "void" for the first voxel cube cut from the digital core model (clockwise, the excursion parameter is $0,0.2,0.3,0.4$ )..

Histograms of the normalized radiodensity distribution for core samples show that the number of voxels with values greater than 0.6 for all samples is smaller than other values. It was expected that the topological characteristics obtained with these values of the excursion parameter will be negligibly small (Fig. 5). 

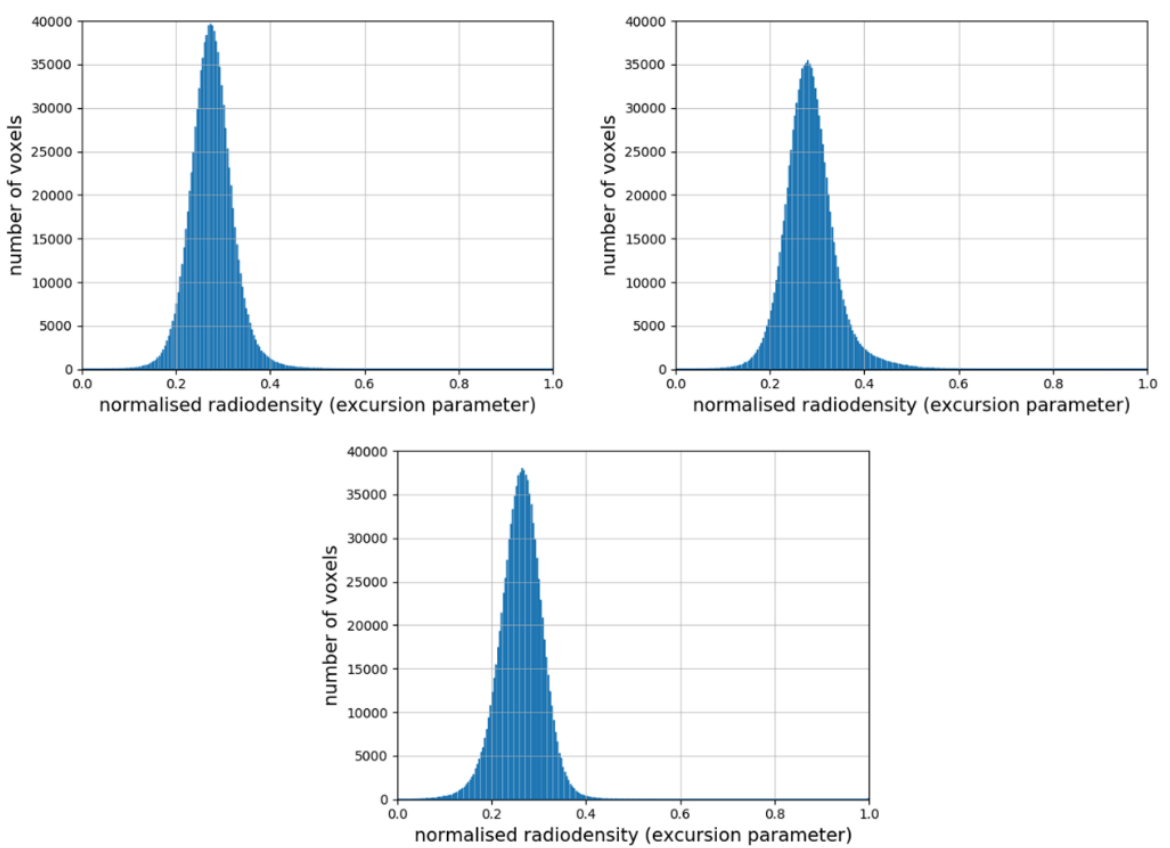

Fig. 5 Histograms of the normalized radiodensity distribution for core samples (clockwise 1 st, 2nd and 3rd cube).

That is because if the excursion parameter is greater than 0.6, voxels marked as "rock skeleton" belong to local consolidations with the highest density values (Fig 6.).

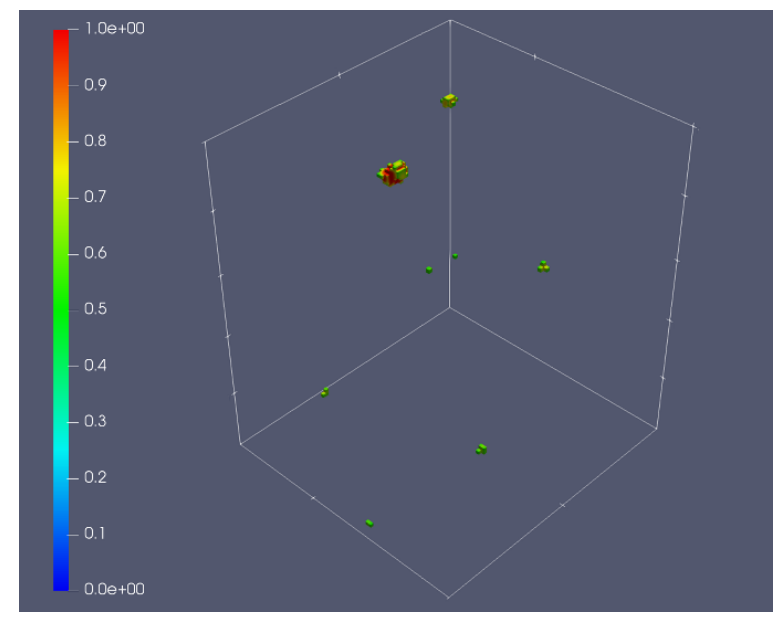

Fig. 6. Local consolidations inside the digital core sample. 
Also these histograms show the difference between second cube dispersion:

$$
\mathrm{D}_{2 \text { nd cube }}=2.86 * 10^{-3}
$$

and first, and third cube dispersion:

$$
\mathrm{D}_{1 \text { st cube }}=\mathrm{D}_{3 \mathrm{rd} \text { cube }}=2.15^{*} 10^{-3}
$$

\subsection{Topological Characteristics of digital core models evaluation}

Topological characteristics of three-dimensional digital core models in the paper are evaluated similarly to topological characteristics of digital geological models in [3].

As expected from histograms (Fig .5), the topological characteristics barely change if the excursion parameter is greater than 0.6. That is because the topological characteristics are close to constant while the excursion parameter is increasing (Fig. 7).
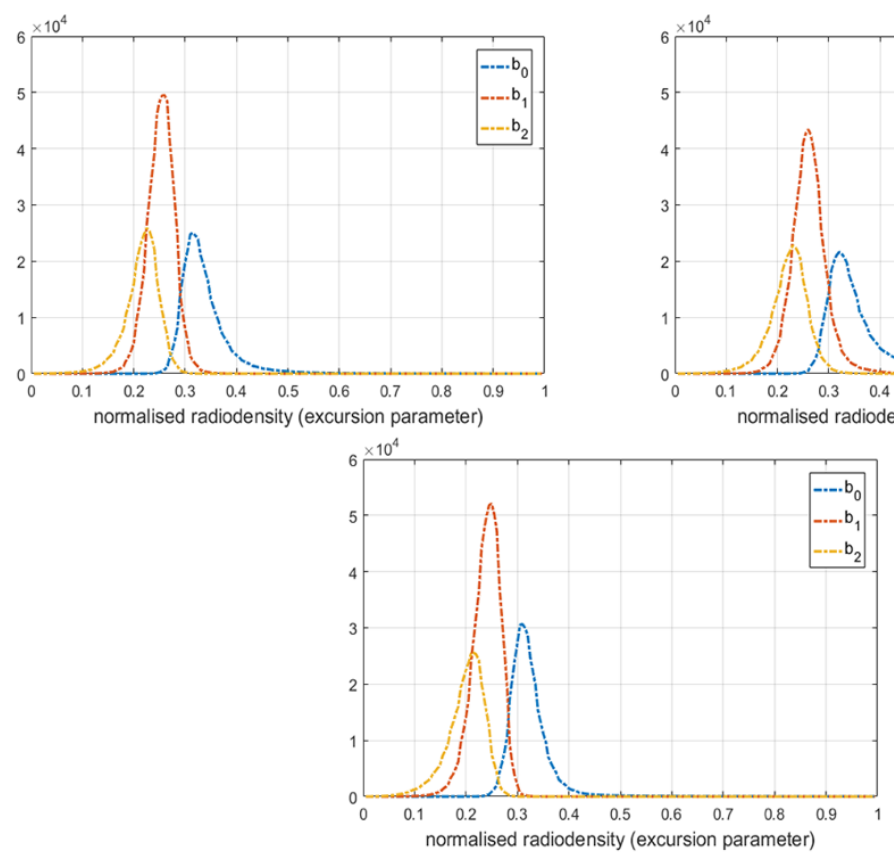

Fig. 7. Relationship between the Betti numbers and the excursion parameter (clockwise - 1st, 2nd and 3rd cube).

The calcualted Betti numbers allow to consider digital core models as realizations of random fields - the same way as stochastic digital geological models [3]. It is shown on a 3-D plot of topological characteristics which axes are $\boldsymbol{b}_{\boldsymbol{0}}, \boldsymbol{b}_{\boldsymbol{1}}$, and $\boldsymbol{b}_{2}$, and the parameter is the excursion (Fig. 8). Shape of the obtained "curves" for the first and the third cube is similar to a "curve", obtained for the Gaussian variogram realization of a geological random field (Fig. 9). 
Analogously for the second cube - shape of the obtained "curve" is similar to a "curve", obtained for the exponential variogram realization of a geological random field (Fig. 9).

In both cases variogram radiuses are little in comparsion of linear size of a model: variogram radius does not exceed $2 \%$ of linear size of a model. It shows a high compartmentalization of these models.
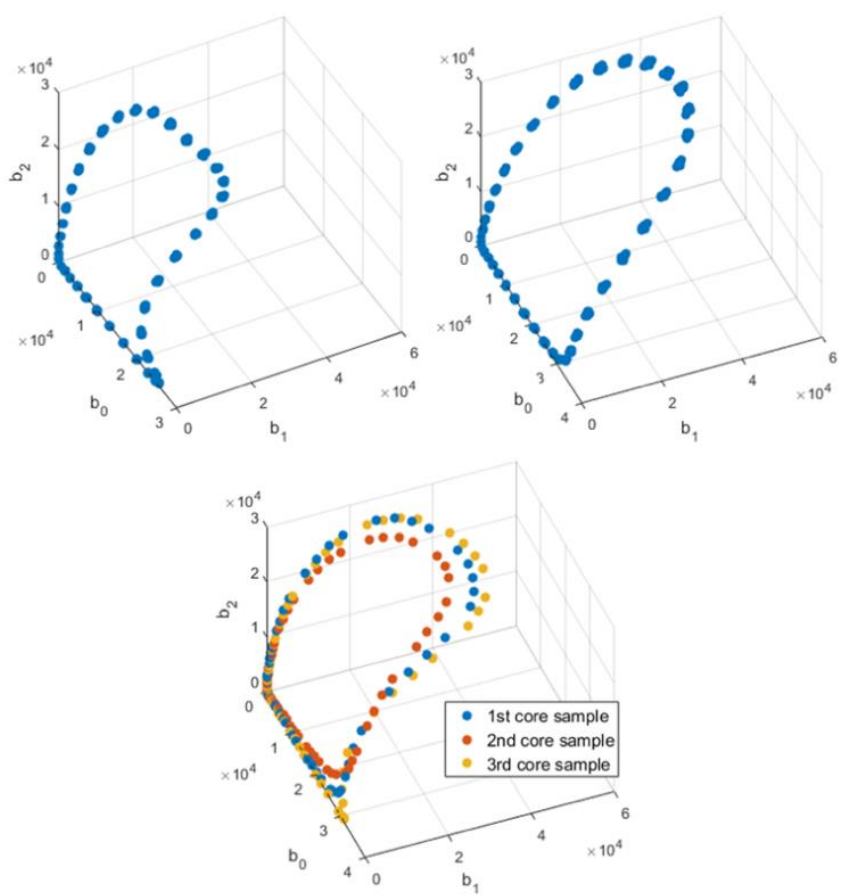

Fig. 8. Topological characteristics in the Betti number axes (clockwise - digital geological model with a Gaussian variogram, digital geological model with an exponential variogram, digital core).
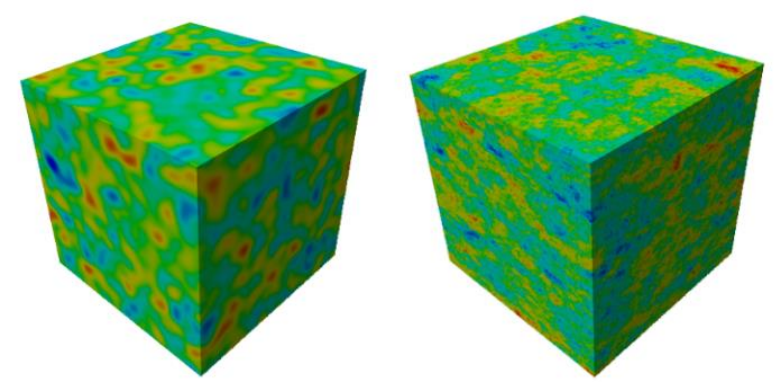

Fig. 9. Realization of a geological random field with a Gaussian (left) and exponential (right) variograms. 
The differences obtained in these "curves" shape indicate an internal core heterogeneity. This difference in the inner complexity of the second cube from the first and third can be caused by an acid treatment carried out on this core sample.

\section{Conclusion}

Analysis of topological characteristics of digital core models allows to:

- find regions of internal core heterogeneity;

- consider digital core models as realizations of digital geological stochastic models. That is how a developed geostatistics methodology can be used in digital core analysis;

The inner complexity and the Betti numbers dependencies for digital core models are going to be researched with a larger amount of cut cubes. The assessment by a specialist is required in terms of their inner structure - porosity, the presence of cracks and caverns.

\section{References}

1. Hasanov M.M., Belozerov B.V., Bochkov A.S., Fuks O.M., Tengelidi D.I.: Automation of lithological-facies analysis on the basis of spectral theory. Publishing House "Neftyanoe Khozyaystvo" (Oil Industry) 12, 48-51 (2015). (Russian)

2. Bazaikin Y.V., Baikov V.A., Taimanov I.A., Yakovlev A.A.: Numerical analysis of topological characteristics of three-dimensional geological models of oil and gas fields. Mathematical Modeling vol. 25, 10, 19-31. (2013). (Russian)

3. Baikov V.A., Gilmanov R.R., Taimanov I.A., Yakovlev A.A.: Topological Characteristics of Oil and Gas Reservoirs and Their Applications. In: Holzinger A., Goebel R., Ferri M., Palade V. (eds) Towards Integrative Machine Learning and Knowledge Extraction. Lecture Notes in Computer Science, vol 10344. Springer, Cham (2017).

4. Krivoshchekov S.N., Kochnev A.A.: Determination of reservoir properties of reservoir rocks using x-ray imaging core. Master's journal. 1, 120-128 (2014). (Russian)

5. Wellington S.L., Vinegar H.J.: X-ray computerized tomography. J. Petrol. Tech., 1987, v. 39, p. $885-898$

6. Yakushina O.A., Ozhogina E.G., Hozyainov M.S.: X-ray computational microtomography - non-destructive method of structural and phase analysis. Computational world (Mir izmereniy) 10, 12-17 (2003). (Russian)

7. Ivanov M.K., Burlin U.K, Kalmykov G.A., Karniushina E.E., Korobova N.I.: Petrophysical methods of core material research. MSU Publishing, Moscow (2008).

8. Zhukovskaia E.A., Lopushniak U.M. The use of X-ray tomography in the study of terrigenous and carbonate reservoirs. Nauchno-technicheskiy vestnik OAO "NK Rosneft" 1, 125 (2008). (Russian)

9. De Vos, W.; Casselman, J.; Swennen, G.R.J. (June 2009). "Cone-beam computerized tomography (CBCT) imaging of the oral and maxillofacial region: A systematic re-view of the literature". International Journal of Oral and Maxillofacial Surgery. 38 (6): 609-625.

10. Baikov, V.A., Bakirov, N.K., Yakovlev, A.A.: Mathematical Geology. I. Introduction to Geostatistics. Izhevsk Institute of Computer Sciences, Izhevsk (2012). (Russian) 